\title{
A multicentre study of hospital staff attitudes to homeless patients
}

\author{
Authors: Rose Glennerster, ${ }^{1}$ Felicity Cooksey, ${ }^{2}$ Viviene Barrow, ${ }^{3}$ Andiran Anduvan, ${ }^{4}$ Kitty Atcheson, ${ }^{4}$ \\ Sarah Kyle ${ }^{5}$ and Philippa Medcalf ${ }^{4}$
}

\section{Aims}

Our aim was to assess attitudes of hospital staff to the management of homeless patients.

\section{Methods}

An online questionnaire was sent to all hospital staff across four regional centres enquiring about their experience of homeless patients, their knowledge of appropriate management and their attitudes to homeless patients. The online questionnaire was advertised through email and on trust homepages. In addition, paper copies of the questionnaire were distributed in each of the regional centres. Two of the centres have homeless healthcare teams (HHTs) (Brighton and Gloucester, 117 responses) and two do not (Bristol and Newcastle, 156 responses). HHTs comprised a minimum of a housing officer, dedicated to ensuring that a safe discharge solution is found.

\section{Results}

Staff in the two hospitals with HHTs encountered more homeless patients on a weekly basis (53\% of staff in Brighton and $37 \%$ of staff in Gloucester) than staff in the two hospitals that did not (16\% in Bristol and 15\% in Newcastle). In three out of four cases, the number of encounters with homeless patients was a strong predictor of positive staff attitudes. Newcastle was the exception as, despite encountering homeless patients the least, $73 \%$ of staff considered the hospital to have a responsibility to address the patients' lack of housing. This was second only to Brighton (83\%) in terms of positive staff attitudes.

The most positive staff attitudes were observed in Brighton, where the HHT comprises a full multidisciplinary team who carry out weekly ward rounds of homeless inpatients, work closely with local services and engage in educating staff and students about homeless healthcare.

Authors: ${ }^{1}$ Brighton and Sussex University Hospitals NHS Trust, Brighton, UK; ${ }^{2}$ Royal United Hospitals Bath NHS Trust, Bath, UK; ${ }^{3}$ Newcastle upon Tyne Hospitals NHS Trust, Newcastle, UK; ${ }^{4}$ Gloucestershire Hospitals NHS Trust, Gloucester, UK; ${ }^{5}$ North Bristol NHS Trust, Bristol, UK

\section{Conclusions}

The attitudes of hospital staff are likely to be influenced by multiple factors, including exposure to homeless patients and having a dedicated HHT. We recommend further education of both staff and students, particularly regarding the role of the HHTs, hospital discharge protocols and ongoing promotion of positive attitudes towards homeless patients.

\section{Conflict of interest statement}

None to declare. 